\title{
Developing Sensitive Golden Plate Biosensor for Early Detection of Serum Cardiac Troponin I as Diagnostic Biomarkers
}

\section{Waleed M. AL-Shawafi ${ }^{1,2,3}$, Numan Salah ${ }^{2,4}$, Taha A. Kumosani ${ }^{1,5,6}$, Ehab M. M. Ali1 ${ }^{1,7}$, , Wesam H.Abdulaal ${ }^{1}$, Abdulrahman L. Al-

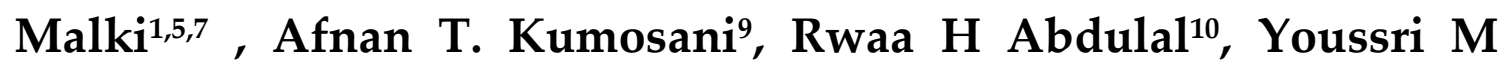 Ahmed ${ }^{1,5,6,11}$, Ibrahim H. Ibrahim ${ }^{1,12}$, Ahmed H. Hammad ${ }^{2}$, M.Sh Abdel-wahab ${ }^{2}$ and Said S. Moselhy $y^{1,5,7,13^{*}}$}

${ }^{1}$ Departmentt of Biochemistry, Faculty of Sciences, King Abdulaziz University, Jeddah, Saudi Arabia.

${ }^{2}$ Centre of Nanotechnology, King Abdulaziz University, Jeddah, Saudi Arabia. ${ }^{3}$ Department of Chemistry, Faculty of Applied Sciences, Taiz University, Taiz, Yemen.

${ }^{4}$ Departmentt. Electrical and Computer Engineering, King Abdulaziz University, Jeddah, Saudi Arabia.

${ }^{5}$ Experimental Biochemistry Unit, King Fahd Medical Research Center, King Abdulaziz University, Jeddah, Saudi Arabia.

${ }^{6}$ Production of Bioproducts for Industrial Applications Research Group, KAU. ${ }^{7}$ Bioactive Natural Products Research Group, King Abdulaziz University, Jeddah, Saudi

${ }^{8}$ Chemistry Department Biochemistry Division, Faculty of Science, Tanta University, Tanta, Egypt Arabia.

${ }^{9}$ Department of pathology and Laboratory Medicine, Blood Bank Transfusion Medicine, King Abdulaziz Medical city, Jeddah, National Guards, , Jeddah, 21423.

${ }^{10}$ Deapertment of medical laboratory Science,, faculty of medical sciences,

Microbiology, Fakeeh Collage for medical scinces, Jeddah, Saudi Arabia

${ }^{11}$ Microbial Biotechnology Dept., National Research Center, Dokki - Cairo, Egypt.

${ }^{12}$ Physics Department (Biophysics Division), Faculty of Science, Ain Shams University, Cairo, Egypt.

${ }^{13}$ Biochemistry Department, Faculty of Science, Ain Shams University, Cairo, Egypt.

*Corresponding author email: moselhy6@hotmail.com 


\title{
Developing Sensitive Golden coated Biosensor for Early Detection of Serum Cardiac Troponin I as Diagnostic Biomarkers
}

\begin{abstract}
Rapid diagnosis of acute myocardial infarction is critical for intervention and treatment to decrease morbidity and mortality. For this reasons, this study aimed to design a sensitive biosensor for cardiac troponin I (cTnI) for early prediction and intervention. The sensor was designed from golden plate immobilized with anti- cTnI antibody conjugated with horseradish peroxidase (HRP) enzyme. The rate of degradation of $\mathrm{H}_{2} \mathrm{O}_{2}$ is directly related to serum cTnI level. Serum cTnI level was quantified from 3 studied groups including; healthy subjects and patients with ischemic heart (IHD) diseases and myocardial infarction (MI) by ELISA and biosensor techniques to achieve the improved sensitivity of the biosensor. It was found that $\mathrm{cTnI}$ levels in healthy subjects ranged from 8-13 ng/dl and in patients with IHD ranged from 20 - $41 \mathrm{ng} / \mathrm{dl}$ and MI range from 57- $180 \mathrm{ng} / \mathrm{dl}$. The sensitivity of biosensor was ranged from $85-95 \%$ compared with ELISA technique. The developed biosensor is promising in ICU hospitals for early diagnosis and prevent complications of the stroke. It is recommended to apply this biosensor in the large scale of patients with different stages of the cardiac disease. .
\end{abstract}

Keywords: Troponin I- Myocardial infarction- Ischemic heart diseases- Biosensors- golden plate

\section{Introduction}

Coronary heart disease (CHD) happens when lipids accumulates on blood vessels leads to decrease of elasticity of vessels and formation of clots that obstruct the flow of blood to the heart muscle. CHD causes angina pectoris, myocardial infarction, or heart attack. Accurate and exact diagnosis of CHD can help cardiologist for making decision to fast intervene and control the progress of disease 
leading to better management. Serum cardiac biomarkers can help in early diagnosis and prognosis to differentiate between coronary heart disease and chest pain, of these markers, troponin I, CK-MB2 and myoglobin are the most routine tests used in the hospital. Three types of Troponins (troponin $\mathrm{C}$, troponin $\mathrm{T}$, and troponin I) are found in cardiac muscles that contribute to muscle contraction. The total creatine kinase $(\mathrm{CK})$ activity is a biomarker of myocyte damage in $\mathrm{MI}$ and in severe muscle breakdown, dystrophy, autoimmune myositides, and acute kidney injury. Three isoforms of the enzyme CK1 (MM), CK2 (MB) and CK3 (BB) are found predominantly in skeletal, heart and brain muscles. The CK2 has been used as an indication for myocardial damage in CHD. In addition, the sensitivity of serum troponin I level assessment replaces CK2 level in most cases. Myoglobin is released from the blood into the urine in muscle injuries. Troponin I level increases in patients with tachyarrhythmias, subarachnoid hemorrhage, myocardial contusion, myocarditis and hypertension $[1,2,3]$.

Enzyme-linked immunosorbent (ELISA) and fluorometric assays are techniques used for detection of biomarkers for CHD. These techniques are time-consuming and qualified personnel. The biosensor made up of a transducer and a biological element that made of an antibody and enzyme. Immunosensors are one of biomedical application of biosensors that antibodies have high affinity towards 
specific antigens. Fabrication of biosensors and immobilization of enzymes to the golden plate are based on the biomedical application [4]. Biosensors established an electric measurement relevant to biochemical and molecular recognition for the desired selective biomarker of interest [4].

Gold plate biosensors (GPB) are being used for biomedical applications, including the targeted delivery of drugs, laser phototherapy of cancer cells and biosensors of immunoassays the detection of nucleotide sequences, DNA and immunodiagnostics of Alzheimer's disease [5-10].

The fabrication of activated gold nanoparticles bind to activated substance to obtained monolayer of gold nanoparticle to bind with ligand on their surface [5]. The ligand was used as anti-thyroxin and immobilized with surface plasmon resonance that bind with tracer of thyroxin [6]. Also, amyloid- $\beta$ derived diffusible ligands (ADDLs), and specific anti-ADDL antibodies was used to be localized in specific optical biosensor to detect the Alzheimer's disease [7]. A golden nanoparticle used to detect specific DNA by using the surface immobilized probe DNA and the metal nanoparticle coated reporter DNA [8]. Eectrocatalytic device containing gold nanoparticles coupled with an electrotrans ducing platform/sensor is achieved to determine the specific identification of tumor cells [9]. The application of gold nanoparticle conjugates in biomedical diagnostics 
and analytics, photothermal and photodynamic therapies, as a carrier for delivering target molecules, and on the immunological and toxicological properties [10].

In this study, golden plate immobilized with anti-cTnI antibody conjugated with horseradish peroxidase (HRP) enzyme was designed for early detection of serum cTnI. The designed golden plate biosensor was tested for serum cTnI level in healthy subjects and patients with IHD diseases and myocardial infarction MI with necrosis and compared results with ELISA techniques. The prepared golden plate binding with antibodies may be promising for marketing.

\section{Materials and methods}

\subsection{Preparation of golden plate}

Gold nanoparticles with purity $99.99 \%$ were used for depositing thin films. The golden nanoparticles (GNP)-Glass substrate was prepared by the pulsed laser deposition (PLD) technique using Neocera Pioneer 180, Neocera, USA. An excimer Laser with KrF gas as a laser source was used [11]. Glass slide was cut to a rectangular shape $(1 \times 2 \mathrm{~cm})$ and ultrasonicated in ethanol and deionized water for 30 min respectively. After drying slide, it was fixed inside the PLD chamber with a distance of around $10 \mathrm{~cm}$. The chamber was evacuated to a base pressure of 2.2.x10-6 torr. The films were deposited at a laser power of $250 \mathrm{~mJ}$ (at $21.2 \mathrm{keV}$ ) at room 
temperature. Around 6000 pulses were used for depositing each film. To obtain a uniform thickness slide was rotated during the deposition at a speed $50 \mathrm{deg} / \mathrm{sec}$. This deposition was carried out in the vacuum without inserting any other gas. The prepared gold coated on glass biosensors (GCB) was then thoroughly rinsed with water. Then, GCB surface was treated with $20 \mathrm{mM}$ cystamine dihydrochloride for $30 \mathrm{~min}$ at room temperature. Cystamine was adsorbed on the exposed patterns of the gold surface and protein (anti-troponin I) binding covalently attached to the amino groups of cystamine. After rinsing with deionized water, $4 \%$ glutaraldehyde solution was treated to the surface of GCB for $30 \mathrm{~min}$ at room temperature, to introduce aldehyde residue, which can bind amine residue of the protein surface.

2.2. Characterization of prepared golden plate

The morphology and microstructures of the deposited GNP in these films were analyzed with the field emission scanning electron microscopy (SEM) (FESEM, JSM-7500F, JEOL, Japan) and field emission transmission electron microscope (TEM) (FETEM, JEM 2100F, JEOL, Japan). The plates were also analyzed by tapping mode atomic force microscopy (AFM). The thickness of the deposited films was measured by SEM cross-section imaging and using the surface profiler, DEKTAK 150 Veeco, UK.

2.3. Preparation of antibody-enzyme conjugates 
$100 \mu \mathrm{l}$ of anti-cTn I monoclonal antibody (mAB) (purchased from Meridian Life Science (ME, USA) was completed to $10 \mathrm{ml}$ of 25 $\mathrm{mM}$ Tris buffer with $0.15 \mathrm{M}$ sodium chloride, $\mathrm{pH}$ 7.2. The conjugation between horseradish peroxidase HRP $(0.132 \mathrm{mg} / \mathrm{ml})$ and anti-troponin $\mathrm{mAb}$ was performed by using HRP conjugation kit (Pierce ${ }^{\circledR}$ Conjugate Purification Kit-44920, Thermo Fisher Scientific Inc).

\subsection{Subjects}

Format consent was taken from all subjects before the start of the study.

Eighteen serum samples were collected from cardiology Unit, King Abdul-Aziz Hospital categorized equally into 3 groups: Group I: Normal, age range 30-55 years, free from any systematic diseases such as diabetic, hypertension, CVD, renal or respiratory. Group II: Male subjects range 32-65 years, suffered from ischemic heart disease. Group III: Male subjects range 31-70 years, suffered from myocardial infarction.

2.5. Measurements of serum cTnI by GCB and ELISA techniques

Serum cTnI level was measured by ELISA technique by using commercial kits purchased from Abcam Company. Antibodies of cTnI are immobilized in the polystyrene well. $50 \mu \mathrm{l}$ sample or standard (troponin I) and $50 \mu \mathrm{l}$ of conjugated anti cTnI were added to the wells. After one hour incubation, the wells were washed. $100 \mu \mathrm{l}$ of Tetramethylbenzidine (TMB) substrate was added and during 10 
min incubation is catalyzed by HRP, generating blue coloration. This reaction was then stopped by adding $100 \mu$ Stop Solution The intensity of color was measured at $450 \mathrm{~nm}$.

Serum cTnI level was measured by prepared GCB technique. $100-\mu l$ of prepared antibody solution was added at room temperature for $5 \mathrm{~min}$ to GCB in Petri dish, washed and blocked with 5\% albumin. $100-\mu l$ of serum sample was added to the GCB then gently shakes the Petri dish and set at room temperature for $15 \mathrm{~min}$. After washing with Tris-saline buffer, $2 \mathrm{ml}$ of prepared an HRP conjugated anti-troponin $\mathrm{mAb}$ was added and shake for two hours. $10 \mu \mathrm{L}$ of $8 \mathrm{mM} \mathrm{H}_{2} \mathrm{O}_{2}$ and $900-\mu \mathrm{lTris}$ were added and incubated for $15 \mathrm{~min}$, and then $80 \mu \mathrm{L}$ of 40 $\mathrm{mM}$ guaiacol was added and read by spectrophotometer at $420 \mathrm{~nm}$ [12].

\section{Results}

The three-dimensional microstructure of golden plate biosensors (GPB) was observed by SEM (Fig 1a) and TEM (fig 1b,c) showed uniform thickness film with $94 \mathrm{~nm}$ layer with the absence of mesoporosity that indicated high quality, more efficient plate bind with primary antibody of troponin I o on their surface. The results of AFM images illustrated the surface morphology and roughness of gold-coated glass before and after treatment with cystamine and glutaraldehyde (Fig 2 a and b) respectively. The surface roughness of the GNP is the distribution of surface heights. The roughness 
increased after GNP treated with cystamine and glutaraldehyde. The actual shape of a surface is effective by surface position and surface heights. It is well known that the melting point of the gold nanoparticles decreases rapidly with decreasing particle size. The treatment of the gold nanoparticles by using cystamine and glutaraldehyde form of spherolytic and hummock-like structures in the gold layers that may be connected with an enhanced diffusion of gold particles at elevated temperature and their aggregation into larger structures.

Analysis of serum troponin I from different patients suffered from cardiac diseases and control subjected by GPB were compared with ELISA results. It was found that serum cTnI levels in healthy subjects ranged from 8-13 ng/dl while in patients with IHD ranged from 20-41 $\mathrm{ng} / \mathrm{dl}$ and MI ranged from 57-180 ng/dl by using two ELISA and GCP techniques. The data obtained from GPB showed high sensitivity and close to results obtained from ELISA Fig (3).

The storage stability of GPB was investigated at different conditions. The GPB was kept at $4{ }^{\circ} \mathrm{C}$ and then tested for detection of serum troponin I in different samples daily for the activity. The results obtained showed that there were no significant changes observed within one week. 


\section{Discussion}

The early noninvasive sensitive cardiac biomarker is critically important in ICU of hospitals. The routine methodology used is ELISA techniques. However, the one-step biosensors can simplify the whole process with high sensitivity. In the current study, we have designed a simple, sensitive gold plate biosensor (GPB) for detection of serum cTnI in patients with CHD. Meanwhile, the fabrication of GPB was being controlled by the anti-troponin antibody, developing color, conjugation of antibody with HRP. The biosensor was developed by classic methodology [13]. The complex of antibody labeled with the signal generating enzyme was captured by cTnI and reaction between HRP and $\mathrm{H}_{2} \mathrm{O}_{2}$ as the indication for plasma cTnI.

The GNPs binding with antibodies and labeled with HRP generates a colored product after time incubation to detect the target molecule qualitatively [14,15]. The GPB was designed by using the uniform thin layer of golden nanoparticles. Characterization of GPB was performed by using TEM and SEM techniques showed no mesoporosity with sizes ranging from 10-50 nm. The previous study reported that surface morphology of GPB by using pulse laser deposition structures does not depend significantly on times but depending on the size particles $[16,17]$.

The GPB was treated with cystamine to get terminal amino groups reacted with the aldehyde groups to yield imine bonds with 
HRP. Cystamine also has -SH group that GNPs were absorbed ontoSH group. However, the surface of GPB morphology was changed when treated with cystamine and glutaraldehyde $[18,19,20]$.

Antibodies $(\mathrm{Ab})$ are protein receptors for biosensing due to their specificity towards their specific antigen. The specificity between the $\mathrm{Ab}$ and antigen interaction has been applied in GPB for clinical diagnosis. Peptides and enzymes are also receptors for GNPs based sensing as they can be functionalized to the surface of GNPs without losing its activity for recognition [21,22]. We investigated the storage stability of the GCB with mAb-cTI, there were no significant changes in results observed within one week. Herein, we investigated the accuracy of the cTnI biosensor by comparing the results by determining cTnI in human serum and with ELISA. As presented healthy normal and patients with IHD and MI were performed with two different techniques, sensitivity is ranged from $85-95 \%$. The sensitivity of results obtained by GPB was compared with results of ELISA method. It was found that the values of serum CTI were very close to that obtained by ELISA. On the hand, standard troponin tested with GPB showed recovery with $97.5 \%$. The results of serum troponin I obtained from GPB showed high reliability and close to results obtained from ELISA techniques.

\section{Conclusion}


The developed biosensor is promising for early diagnosis and fast intervention for management of $\mathrm{CHD}$. This will decrease the mortality of patients with cardiac disease due to late diagnosis.

\section{Ethical approval}

All procedures performed in studies involving human participants were in accordance with the ethical approval \# (12-CEGMR-2-ETH), King Abdulaziz University.

\section{Author Contributions:}

SSM, TAK, EMA, YMS, IHI and AAL conceived and designed the experimental protocols. WMA, NS, AHA, MSH,ATK and RA designed and performed the experiments, analyzed the data, and wrote the paper. All authors contributed to the writing of the manuscript.

\section{Conflict of interest}

The authors declare that they have no conflict of interest.

\section{References}

[1].Roffi M, Patrono C, Collet JP, et al. ESC Guidelines for the management of acute coronary syndromes in patients presenting without persistent ST-segment elevation: Task Force for the Management of Acute Coronary Syndromes in Patients Presenting without Persistent ST-Segment Elevation of the European Society of Cardiology (ESC). Eur Heart J. 2016;37:267-15.

[2]. Zimmerman J, Fromm R, Meyer D et al. Diagnostic marker cooperative study for the diagnosis of myocardial infarction. Circulation. 1999;99:1671-77. 
[3].Sandhu R, Aronow WS, Rajdev A, et al. Relation of cardiac troponin I level with in-hospital mortality in patients with ischemic stroke, intracerebral hemorrhage, and subarachnoid hemorrhage. Am J Cardiol. 2008;102:632-34

[4].Mehrotra P. Biosensors and their applications - A review. Journal of oral biology and craniofacial research. 2016;153-59

[5].Nath N, Chilkoti A. Label-Free Colorimetric Biosensing Using Nanoparticles J. Fluorescence. 2004; 14:377-89.

[6].Adamczyk M, Moore JA and Yu Z. Application of surface plasmon resonance toward studies of low-molecular-weight antigen-antibody binding interactions.Methods. 2000; 20:319-28.

[7].Haes AJ, Chang L, Klein WL, et al. Detection of a biomarker for Alzheimer's disease from synthetic and clinical samples using a nanoscale optical biosensor. J Am Chem Soc. 2005; 127:2264-71.

[8].Peng HI, Miller BL. Recent advancements in optical DNA biosensors: Exploiting the plasmonic effects of metal nanoparticles. Analyst. 2011; 136:43647.

[9]. de la Escosura MA, Sanchez EC, Diaz FB, et al. Rapid identification and quantification of tumor cells using an electrocatalytic method based on gold nanoparticles Anal. Chem. 2009; 81:10268-74.

[10].Dykman LA, Khlebtsov NG. Gold nanoparticles in biology and medicine: recent advances and prospects. Acta Naturae. 2010; 3:34-55.

[11].Numan S, Alshahrie A, Iqbal J, et al. Tribological behavior of diamond-like carbon thin films deposited by the pulse laser technique at different substrate temperatures. Tribology International.2016; 103:274-280.

[12]. Kherd AA, Helmi N, Balamash KS, etal., Changes in erythrocyte ATPase activity under different pathological conditions. Afr Health Sci. 2017 Dec;17(4):1204-1210. doi: 10.4314/ahs.v17i4.31.

[13].Ahammad AJ, Choi $\mathrm{YH}, \mathrm{Koh} \mathrm{K}$, et al. Electrochemical detection of cardiac biomarker troponin I at gold nanoparticle-modified ITO electrode by using open circuit potential. Int. J Electrochem Sci; 2011: 1906-16. 
[14].Qin C, Wen $\mathrm{W}$, Zhang $\mathrm{XH}$, et al. Visual detection of thrombin using a strip biosensor through aptamer-cleavage reaction with enzyme catalytic amplification. Analyst 2015; 140:7710-17.

[15]. Chapman R, Lin Y, Burnapp M, et al. Multivalent nanoparticle networks enable point-of-care detection of human phospholipase-A2 in serum. ACS Nano 2015; 9:2565-73.

[16].Robertson JW, Cai M and Pemberton JE. Insulating ultrathin silica films formed by a room-temperature sol-gel process. Advanced Materials. 2001; 13:662-67.

[17].Sunil SA and Vinod CP. Gold incorporated mesoporous silica thin film model surface as a robust user and the catalytically active substrate. Molecules 2016;667:1-12

[18].Roduner E. Size matters: Why nanomaterials are different. Chem Soc Rev 2006; 35: 583-92.

[19].Kan C, Zhu X and Wang G. Single-crystalline gold microplates: synthesis, characterization, and thermal stability. J Phy ChemB 2006; 110:4651-56.

[20].Liu HB , Ascencio JA, Perez A M, et al. Melting behavior of nanometersized gold isomers. Surface Sci 2001; 491:88-98.

[21].Tighe PJ, Ryder RR, Todd I, et al. ELISA in the multiplex era: Potentials and pitfalls. Proteom. Clin Appl 2015; 9: 406-422.

[22].Cordeiro M, Carlos FF, Pedrosa P, et al. Gold Nanoparticles for Diagnostics: Advances towards Points of Care. Diagnostics 2016; 6:1-20 